\title{
DENJOY-BOCHNER ALMOST PERIODIC FUNCTIONS
}

\author{
B. K. PAL and S. N. MUKHOPADHYAY
}

(Received 15 July 1981)

Communicated by E. Strzelecki

\begin{abstract}
The special Denjoy-Bochner integral (the $D^{*} B$-integral) which are generalisations of Lebesgue-Bochner integral are discussed in $[7,6,5]$. Just as the concept of numerical almost periodicity was extended by Burkill [3] to numerically valued $\mathrm{D}^{*}$ - or $\mathrm{D}$-integrable function, we extend the concept of almost periodicity for Banach valued function to Banach valued $D^{*} B$-integrable function. For this purpose we introduce as in [3] a distance in the space of all $D^{*} B$-integrable functions with respect to which the $\mathrm{D}^{*} \mathrm{~B}$-almost periodicity is defined. It is shown that the $\mathrm{D}^{*} \mathrm{~B}$-almost periodicity shares many of the known properties of the almost periodic Banach valued function $[1,4]$.
\end{abstract}

1980 Mathematics subject classification (Amer. Math. Soc.): 26 A 99, 43 A 60.

\section{Definitions and terminology}

For the definition of almost periodicity for numerical valued and Banach valued functions we refer to [2] and [1,4] respectively. Throughout the paper $\mathbf{R}$ and $\mathbf{C}$ will denote the real line and the complex plane and $\mathbf{X}$ will denote a fixed complex Banach space with norm $\|\cdot\|$. For a function $f$ defined on $\mathbf{R}, f_{\eta}$ will denote the translation of $f$ by the number $\eta$; that is, $f_{\eta}(x)=f(x+\eta)$.

Definition 1.1 [3]. Let $\mathscr{Q}^{*}$ be the class of all functions $f: \mathbf{R} \rightarrow \mathbf{C}$ such that $f$ is $\mathrm{D}^{*}$-integrable on each closed interval $[a, b] \subset \mathbf{R}$. For $f, g \in \mathbb{Q}^{*}$ the $\mathrm{D}^{*}$ distance

(C) 1984 Australian Mathematical Society 0263-6115/84\$A2.00+0.00 
between $f$ and $g$ is defined to be

$$
\rho_{\mathrm{D}^{*}}(f, g)=\sup _{\substack{0 \leqslant h \leqslant 1 \\-\infty<x<\infty}}\left|\left(\mathrm{D}^{*}\right) \int_{x}^{x+h}\{f(t)-g(t)\} d t\right| .
$$

A function $f \in \mathscr{Q}^{*}$ is almost periodic in the sense of the $\mathrm{D}^{*}$ distance (or simply $D^{*}$ a.p.) if, given $\varepsilon>0$ there is a relatively dense set $\{\tau\}$ such that

$$
\rho_{\mathrm{D}^{*}}\left(f_{\tau}, f\right)<\varepsilon
$$

for all $\tau \in\{\tau\}$.

Definition $1.2[7,6,5]$. A function $f:[a, b] \rightarrow \mathbf{X}$ is said to be special Denjoy-Bochner integrable or $\mathrm{D}^{*} \mathrm{~B}$-integrable in $[a, b]$ if there is a function $F$ : $[a, b] \rightarrow \mathbf{X}$ such that $F$ is strongly $\mathrm{ACG}_{*}$ on $[a, b]$ and $\mathrm{AD}_{\mathrm{s}} F=f$ almost everywhere in $[a, b]$ where $\mathrm{AD}_{\mathrm{s}} F$ stands for the strong approximate derivative of $F$. The function $F$ is then called an indefinite $\mathrm{D}^{*} \mathrm{~B}$-integral of $f$ on $[a, b]$ and $F(b)-F(a)$ is called its definite $\mathrm{D}^{*} \mathrm{~B}$-integral on $[a, b]$ and is denoted by

$$
\left(\mathrm{D}^{*} \mathrm{~B}\right) \int_{a}^{b} f(\xi) d \xi
$$

Definition 1.3. Let $\mathscr{D} * \mathscr{B}$ be the class of all functions $f: \mathbf{R} \rightarrow \mathbf{X}$ such that $f$ is $\mathrm{D}^{*} \mathrm{~B}$-integrable on each closed interval $[a, b] \subset \mathbf{R}$. For $f, g \in \mathbb{Q} * \mathscr{g}$ the $\mathrm{D}^{*} \mathrm{~B}$ distance between $f$ and $g$ is defined to be

$$
\rho_{\mathrm{D}^{*} \mathrm{~B}}(f, g)=\sup _{\substack{0 \leqslant h \leqslant 1 \\-\infty<x<\infty}}\left\|\left(\mathrm{D}^{*} \mathrm{~B}\right) \int_{x}^{x+h}\{f(t)-g(t)\} d t\right\| .
$$

A function $f \in \mathscr{Q}^{*} \mathscr{B}$ is said to be almost periodic in the sense of the $\mathrm{D}^{*} \mathrm{~B}$-distance (or, simply $\mathrm{D}^{*} \mathrm{~B}$ a.p.) if, given $\varepsilon>0$ there is a relatively dense set $\{\tau\}=\{\tau ; f, \varepsilon\}$ such that

$$
\rho_{\mathrm{D}^{* \mathrm{~B}}}\left(f_{\tau}, f\right)<\varepsilon
$$

for all $\tau \in\{\tau\}$. Clearly every almost periodic function $f: \mathbf{R} \rightarrow \mathbf{X}$ is $\mathrm{D}^{*} \mathrm{~B}$ a.p.

Remark. This definition of the $\mathrm{D}^{*} \mathrm{~B}$-distance, of course, does not guarantee that

$$
\rho_{\mathrm{D} * \mathrm{~B}}(f, g)<\infty
$$

for all $f, g \in \mathbb{D} * \mathbb{B}$. We shall, however, prove that every $\mathrm{D}^{*} \mathrm{~B}$ a.p. function $f$ is $\mathrm{D}^{*} \mathrm{~B}$-bounded, that is

$$
\rho_{\mathrm{D}^{* \mathrm{~B}}}[f]=\rho_{\mathrm{D}^{* \mathrm{~B}}}(f, \theta)<\infty
$$


from which it will follow that for all $\mathrm{D}^{*} \mathrm{~B}$ a.p. functions $f$ and $g$

$$
\rho_{\mathrm{D}^{*} \mathrm{~B}}(f, g)<\infty \text {. }
$$

Definition 1.4. A continuous function $\phi: \mathbf{R} \times[0,1] \rightarrow \mathbf{X}$ is called almost periodic in $x \in \mathbf{R}$ uniformly with respect to $h \in[0,1]$ if to arbitrary $\varepsilon>0$ corresponds a relatively dense set $\{\tau\}$ such that

$$
\sup _{\substack{0 \leqslant h \leqslant 1 \\-\infty<x<\infty}}\|\phi(x+\tau, h)-\phi(x, h)\|<\varepsilon
$$

for all $\tau \in\{\tau\}$.

The following result for integration by parts for the $D^{*} B$-integral, which will be needed later, is proved in [5].

Theorem 1.5. Let $f:[a, b] \rightarrow \mathbf{X}$ be $\mathrm{D}^{*} \mathrm{~B}$-integrable and

$$
F(\xi)=\int_{a}^{\xi} f(t) d t .
$$

Let $\mathrm{g}:[a, b] \rightarrow \mathbf{R}$ be L-integrable and let

$$
G(\xi)=\int_{a}^{\xi} g(t) d t .
$$

Then $f G$ is $\mathrm{D}^{*} \mathrm{~B}$-integrable over $[a, b]$ and

$$
\int_{a}^{b} f G=[F G]_{a}^{b}-\int_{a}^{b} F g
$$

\section{Properties of $D^{*} B$ a.p. functions}

THEOREM 2.1. If a function $f$ is $\mathrm{D}^{*} \mathrm{~B}$ a.p. then

$$
F(x)=\int_{0}^{x} f(t) d t
$$

is uniformly continuous.

Since the $\mathrm{D}^{*} \mathrm{~B}$-integral,

$$
F(x)=\int_{0}^{x} f(t) d t
$$

is continuous and since a continuous Banach valued function is uniformly continuous on a closed interval the theorem can be proved by the usual process.

THeOREM 2.2. If $f$ is $\mathrm{D}^{*} \mathrm{~B}$ a.p. then the function $\phi: \mathbf{R} \times[0,1] \rightarrow \mathbf{X}$ defined by

$$
\phi(x, h)=\int_{x}^{x+h} f(t) d t
$$

is almost periodic in $x \in \mathbf{R}$, uniformly with respect to $h \in[0,1]$. 
Proof. We first show that the function $\phi$ is continuous. Let $\varepsilon>0$ be arbitary. Since by Theorem $2.1 F(x)=\int_{0}^{x} f(t) d t$ is uniformly continuous, there is a $\delta>0$ such that $\left\|F\left(x_{1}\right)-F\left(x_{2}\right)\right\|<\varepsilon / 2$ whenever $\left|x_{1}-x_{2}\right|<\delta$ for all $x_{1}, x_{2} \in \mathbf{R}$. Now, let $\left(x_{0}, h_{0}\right) \in \mathbf{R} \times[0,1]$ be arbitrary. Then

$$
\begin{aligned}
\left\|\phi\left(x_{0}, h_{0}\right)-\phi(x, h)\right\| & =\left\|\int_{x_{0}}^{x_{0}+h_{0}} f(t) d t-\int_{x}^{x+h} f(t) d t\right\| \\
& =\left\|F\left(x_{0}+h_{0}\right)-F\left(x_{0}\right)-F(x+h)+F(x)\right\| \\
& \leqslant\left\|F\left(x_{0}+h_{0}\right)-F(x+h)\right\|+\left\|F(x)-F\left(x_{0}\right)\right\| \\
& <\varepsilon / 2+\varepsilon / 2 \\
& =\varepsilon
\end{aligned}
$$

whenever $\left|x-x_{0}\right|<\delta / 2,\left|h-h_{0}\right|<\delta / 2$. Hence $\phi(x, h)$ is continuous on $\mathbf{R} \times$ $[0,1]$.

Now, since $f$ is $\mathrm{D}^{*} \mathrm{~B}$ a.p., corresponding to $\varepsilon>0$ there is a relatively dense set $\{\tau\}$ such that $\rho_{\mathrm{D}^{*} \mathrm{~B}}\left(f_{\tau}, f\right)<\varepsilon$ for all $\tau \in\{\tau\}$. Hence

$$
\sup _{\substack{0 \leqslant h \leqslant 1 \\-\infty<x<\infty}}\left\|\int_{x}^{x+h} f(t+\tau) d t-\int_{x}^{x+h} f(t) d t\right\|<\varepsilon,
$$

that is,

$$
\sup _{\substack{0 \leqslant h \leqslant 1 \\-\infty<x<\infty}}\left\|\int_{x+\tau}^{x+\tau+h} f(t) d t-\int_{x}^{x+h} f(t) d t\right\|<\varepsilon
$$

from which it follows that

$$
\sup _{\substack{0<h \leq 1 \\-\infty<x<\infty}}\|\phi(x+\tau, h)-\phi(x, h)\|<\varepsilon,
$$

which completes the proof.

LEMmA 2.3. Let $\mathcal{C}_{\mathbf{X}}[0,1]$ be the Banach space of all continuous functions $y$ : $[0,1] \rightarrow \mathbf{X}$ with norm

$$
\|y\|_{e_{\mathbf{x}}}=\sup _{0 \leqslant h \leqslant 1}\|y(h)\|
$$

and let $\phi: \mathbf{R} \times[0,1] \rightarrow \mathbf{X}$ be a continuous function. Then the function $\Phi: \mathbf{R} \rightarrow$ $e_{\mathbf{X}}[0,1]$ defined by

$$
\Phi(x)=\phi(x, \cdot)
$$

is almost periodic if and only if the function $\phi$ is almost periodic in $x \in \mathbf{R}$, uniformly with respect to $h \in[0,1]$. 
Proof. Since $\|\Phi(x)\|_{e_{\mathbf{x}}}=\sup _{0 \leqslant h \leqslant 1}\|\phi(x, h)\|$ we have

$$
\|\Phi(x+\tau)-\Phi(x)\| e_{\mathbf{x}}=\sup _{0 \leqslant h \leqslant 1}\|\phi(x+\tau, h)-\phi(x, h)\|
$$

and so the result follows.

LEMma 2.4. If the continuous functions $\phi: \mathbf{R} \times[0,1] \rightarrow \mathbf{X}$ and $\psi: \mathbf{R} \times[0,1] \rightarrow \mathbf{X}$ are almost periodic in $x \in \mathbf{R}$ uniformly with respect to $h \in[0,1]$ then $\phi+\psi$ is so.

Proof. Let $\mathcal{C}_{\mathbf{X}}[0,1]$ be as in Lemma 2.3 and let $\Phi: \mathbf{R} \rightarrow \mathcal{C}_{\mathbf{X}}[0,1]$ and $\Psi$ : $\mathbf{R} \rightarrow \mathcal{C}_{\mathbf{X}}[0,1]$ be defined by

$$
\Phi(x)=\phi(x, \cdot), \quad \Psi(x)=\psi(x, \cdot) .
$$

Then by Lemma 2.3, $\Phi$ and $\Psi$ are almost periodic and so is the sum $\Phi+\Psi$, and hence by Lemma 2.3, $\phi+\psi$ is almost periodic in $x \in \mathbf{R}$ uniformly with respect to $h \in[0,1]$.

TheOREM 2.5. If $f$ and $g$ are $\mathrm{D}^{*} \mathrm{~B}$ a.p. then so is $f+g$.

Proof. By Theorem 2.2, the functions $\phi(x, h)=\int_{x}^{x+h} f(t) d t$ and $\psi(x, h)=$ $\int_{x}^{x+h} g(t) d t$ are almost periodic in $x \in \mathbf{R}$ uniformly with respect to $h \in[0,1]$. Hence by Lemma $2.4, \phi(x, h)+\psi(x, h)$ is almost periodic in $x \in \mathbf{R}$ uniformly with rspect to $h \in[0,1]$. So, given $\varepsilon>0$, there is a relatively dense set $\{\tau\}$ such that

$$
\sup _{\substack{0 \leq h \leq 1 \\-\infty<x<\infty}}\|\phi(x+\tau, h)+\psi(x+\tau, h)-\phi(x, h)-\psi(x, h)\|<\varepsilon
$$

for all $\tau \in\{\tau\}$. Hence

$$
\sup _{\substack{0 \leq h \leq 1 \\-\infty<x<\infty}}\left\|\int_{x+\tau}^{x+\tau+h}\{f(t)+g(t)\} d t-\int_{x}^{x+h}\{f(t)+g(t)\} d t\right\|<\varepsilon,
$$

that is,

$$
\sup _{\substack{0 \leq h<1 \\-\infty<x<\infty}}\left\|\int_{x}^{x+h}[\{f(t+\tau)+g(t+\tau)\}-\{f(t)+g(t)\}] d t\right\|<\varepsilon,
$$

that is,

$$
\rho_{\mathrm{D}^{*} \mathrm{~B}}\left((f+g)_{\tau}, f+g\right)<\varepsilon
$$

for all $\tau \in\{\tau\}$. Hence $f+g$ is $\mathrm{D}^{*} \mathrm{~B}$ a.p.

THeOREM 2.6. If $f$ is $\mathrm{D}^{*} \mathrm{~B}$ a.p. then $f$ is $\mathrm{D}^{*} \mathrm{~B}$ bounded, that is,

$$
\rho_{\mathrm{D}^{* \mathrm{~B}}}[f]=\rho_{\mathrm{D}^{* \mathrm{~B}}}(f, \theta)<\infty .
$$


Proof. Letting $\phi(x, h)=\int_{x}^{x+h} f(t) d t$ and constructing the function $\Phi: \mathbf{R} \rightarrow$ $\bigodot_{\mathbf{X}}[0,1]$ as in Lemma 2.3 we see $\Phi$ is almost periodic. Then by [1, page 5 , property IV], the range of $\Phi$ is relatively compact and hence

$$
\sup _{-\infty<x<\infty}\|\Phi(x)\|_{e_{\mathbf{x}}}<\infty
$$

Hence by the definition of $\|\cdot\|_{e_{\mathbf{x}}}$

$$
\sup _{\substack{0 \leq h \leq 1 \\-\infty<x<\infty}}\|\phi(x, h)\|<\infty
$$

that is,

$$
\sup _{\substack{0 \leq h \leq 1 \\-\infty<x<\infty}}\left\|\int_{x}^{x+h} f(t) d t\right\|<\infty
$$

that is,

$$
\rho_{\mathrm{D}^{*} \mathrm{~B}}(f, \theta)<\infty
$$

THEOREM 2.7. If $f$ is $\mathrm{D}^{*} \mathrm{~B}$ a.p. then $f$ is uniformly continuous with respect to the metric $\rho_{\mathrm{D}^{*} \mathrm{~B}}$; that is, for every $\varepsilon>0$ there is $\delta>0$ such that

$$
\rho_{\mathrm{D}^{* \mathrm{~B}}}\left(f_{\eta}, f\right)<\varepsilon
$$

for all $\eta$ satisfying $|\eta|<\delta$.

Proof. Since $f$ is $\mathrm{D}^{*} \mathrm{~B}$ a.p. by Theorem 2.2 and Lemma 2.3 the function $\Phi$ : $\mathbf{R} \rightarrow \mathcal{C}_{\mathbf{X}}[0,1]$ defined by $\Phi(x)=\phi(x, \cdot)$ is almost periodic, where $\phi(x, h)=$ $\int_{x}^{x+h} f(t) d t$. By [1, page 5, property III], $\Phi$ is uniformly continuous. So, for arbitrary $\varepsilon>0$ there is $\delta>0$ such that

$$
\sup _{-\infty<x<\infty}\|\Phi(x+\eta)-\Phi(x)\| e_{\mathbf{x}}<\varepsilon
$$

for all $\eta$ satisfying $|\eta|<\delta$. That is,

$$
\sup _{\substack{0 \leqslant h \leqslant 1 \\-\infty<x<\infty}}\|\phi(x+\eta, h)-\phi(x, h)\|<\varepsilon,
$$

that is,

$$
\sup _{\substack{0<h<1 \\-\infty<x<\infty}}\left\|\int_{x}^{x+h}\{f(t+\eta)-f(t)\} d t\right\|<\varepsilon,
$$


that is,

$$
\rho_{\mathrm{D}^{*} \mathrm{~B}}\left(f_{\eta}, f\right)<\varepsilon
$$

whenever $|\eta|<\delta$.

THEOREM 2.8. If $\left\{f_{n}\right\}$ is a sequence of $\mathrm{D}^{*} \mathrm{~B}$ a.p. functions such that $f_{n} \rightarrow f$ with respect to the metric $\rho_{\mathrm{D}^{*} \mathrm{~B}}$ then $f$ is $\mathrm{D}^{*} \mathrm{~B}$ a.p.

Proof. Let $\varepsilon>0$ be arbitrary. Then there is $N$ such that $\rho_{\mathrm{D}^{*} \mathrm{~B}}\left(f_{n}, f\right)<\varepsilon / 3$ for all $n \geqslant N$. Since $f_{N}$ is $\mathrm{D}^{*} \mathrm{~B}$ a.p. so there is a relatively dense set $\{\tau\}$ for which $\rho_{\mathrm{D} * \mathrm{~B}}\left(\left(f_{N}\right)_{\tau}, f_{N}\right)<\varepsilon / 3$. Hence

$$
\begin{aligned}
\rho_{\mathrm{D}^{*} \mathrm{~B}}\left(f_{\tau}, f\right) & \leqslant \rho_{\mathrm{D}^{* \mathrm{~B}}}\left(f_{\tau},\left(f_{N}\right)_{\tau}\right)+\rho_{\mathrm{D}^{* \mathrm{~B}}}\left(\left(f_{N}\right)_{\tau}, f_{N}\right)+\rho_{\mathrm{D}^{*} \mathrm{~B}}\left(f_{N}, f\right) \\
& =\rho_{\mathrm{D}^{* \mathrm{~B}}}\left(f, f_{N}\right)+\rho_{\mathrm{D}^{*} \mathrm{~B}}\left(\left(f_{N}\right)_{\tau}, f_{N}\right)+\rho_{\mathrm{D}^{* \mathrm{~B}}}\left(f_{N}, f\right) \\
& <\varepsilon .
\end{aligned}
$$

Thus $f$ is $\mathrm{D}^{*} \mathrm{~B}$ a.p.

THEOREM 2.9. If $f$ is $\mathrm{D}^{*} \mathrm{~B}$ a.p. and $u(x)$ is almost periodic numerical valued function with its derivative $u^{\prime}(x)$ uniformly continuous then $f(x) u(x)$ is $\mathrm{D}^{*} \mathrm{~B}$ a.p.

The proof of the theorem is similar to that of the corresponding theorem of [3]. In fact all the arguments of [3] will apply in this case taking into account the fact that the integration by parts formula for integral is given in Theorem 1.5.

Lemma 2.10. If $f$ is $\mathrm{D}^{*} \mathrm{~B}$ a.p. then $x^{*} f$ is $\mathrm{D}^{*}$ a.p. for every $x^{*} \in \mathbf{X}^{*}$, where $\mathbf{X}^{*}$ is the conjugate space of the Banach space $\mathbf{X}$.

Proof. Take any $x^{*} \in \mathbf{X}^{*}$ and $\varepsilon>0$. Then there corresponds a relatively dense set $\{\tau\}=\left\{\tau ; f, \varepsilon(\|x *\|+1)^{-1}\right\}$ such that

$$
\sup _{\substack{0 \leqslant h \leqslant 1 \\-\infty<x<\infty}}\left\|\int_{x}^{x+h}\{f(t+\tau)-f(t)\} d t\right\|<\varepsilon\left(\left\|x^{*}\right\|+1\right)^{-1}
$$

for all $\tau \in\{\tau\}$. Now since $f$ is $\mathrm{D}^{*} \mathrm{~B}$ a.p., $f$ is $\mathrm{D}^{*} \mathrm{~B}$-integrable on each closed interval $[a, b]$ and so by a result of $[5] x^{*} f$ is $\mathrm{D}^{*}$-integrable on each $[a, b]$ and therefore $x^{*} f \in \mathscr{D}^{*}$. Moreover

$$
x^{*} \int_{x}^{x+h} f(t) d t=\int_{x}^{x+h} x^{*} f(t) d t
$$


for all $x \in \mathbf{R}$ and $h \in[0,1]$. Hence for all $\tau \in\{\tau\}$

$$
\begin{aligned}
\sup _{\substack{0 \leqslant h \leqslant 1 \\
-\infty<x<\infty}} \mid \int_{x}^{x+h}\left\{x^{*} f(t+\tau)-\right. & \left.x^{*} f(t)\right\} d t \mid \\
& =\sup _{\substack{0 \leqslant h \leqslant 1 \\
-\infty<x<\infty}}\left|\int_{x}^{x+h} x^{*}\{f(t+\tau)-f(t)\} d t\right| \\
& =\sup _{\substack{0 \leq h \leq 1 \\
-\infty<x<\infty}}\left|x^{*} \int_{x}^{x+h}\{f(t+\tau)-f(t)\} d t\right| \\
& \leqslant \sup _{\substack{0 \leqslant h \leqslant 1 \\
-\infty<x<\infty}}\left\|x^{*}\right\|\left\|\int_{x}^{x+h}\{f(t+\tau)-f(t)\} d t\right\| \\
& =\left\|x^{*}\right\| \sup _{\substack{-\infty \leqslant h \leqslant 1 \\
-\infty<x<\infty}}\left\|\int_{x}^{x+h}\{f(t+\tau)-f(t)\} d t\right\| \\
& <\left\|x^{*}\right\| \varepsilon\left(\left\|x^{*}\right\|+1\right)^{-1}<\varepsilon .
\end{aligned}
$$

This completes the proof of the lemma.

Lemma 2.11. If $x^{*}$ f is $\mathrm{D}^{*}$ a.p. for all $x^{*} \in \mathbf{X}^{*}$ and if

$$
F(t)=\int_{0}^{t} f(x) d x
$$

is bounded then $F$ is weakly almost periodic (that is, $x^{*} F$ is almost periodic for all $x^{*} \in \mathbf{X}^{*}$ ).

Proof. The function $F(t)$ being bounded $x^{*} F(t)$ is also bounded for all $x^{*} \in \mathbf{X}^{*}$ and since

$$
x^{*} F(t)=\left(\mathrm{D}^{*}\right) \int_{0}^{t} x^{*} f(x) d x,
$$

$x^{*} F$ is almost periodic by [3], that is, $F$ is weakly almost periodic.

THEOREM 2.12. If $f$ is $\mathrm{D}^{*} \mathrm{~B}$ a.p. and if

$$
F(t)=\int_{0}^{t} f(x) d x
$$

is such that the range of $F$ is relatively compact then $F$ is almost periodic.

Proof. By Lemma 2.10, $x^{*} f$ is $\mathrm{D}^{*}$ a.p. for all $x^{*} \in \mathbf{X}^{*}$. The range of $F$ being relatively compact (that is, its closure being compact) $F$ is bounded. Hence by Lemma $2.11, F$ is weakly almost periodic. So by [1, page 45 , property $\mathrm{X}] F$ is almost periodic. 
THEOREM 2.13. The class of all $\mathrm{D}^{*} \mathrm{~B}$ a.p. functions is identical with the $\mathrm{D}^{*} \mathrm{~B}$-closure of the set of all trigonometric polynomials

$$
P(t)=\sum_{r=1}^{n} a_{r} e^{i \lambda_{r} t}
$$

where $a_{r} \in \mathbf{X}, \lambda_{r} \in \mathbf{R}$.

The theorem can be proved in the same way as the corresponding theorem of $D$ a.p. functions of [3].

THeOrem 2.14. If $f$ is $\mathrm{D}^{*} \mathrm{~B}$ a.p. and is uniformly continuous then $f$ is almost periodic.

Proof. Let $\phi: \mathbf{R} \rightarrow \mathbf{R}$ be a nonnegative function with support $[0,1]$ having continuous derivative $\phi^{\prime}$ such that $\int_{0}^{1} \phi(t) d t=1$. For a fixed $n$ let $\phi_{n}(x)=n \phi(n x)$. Then $\phi_{n}$ is a nonnegative function with support $[0,1 / n]$ having continuous derivative $\phi_{n}^{\prime}$ and $\int_{0}^{1 / n} \phi_{n}(t) d t=1$. Let

$$
f_{n}(x)=\int_{0}^{1 / n} f(t+x) \phi_{n}(t) d t .
$$

Then we shall show that $f_{n}$ is almost periodic for each $n$. Let $n$ be fixed and let $\varepsilon>0$ be arbitrary. Let

$$
M=\sup _{0 \leqslant x \leqslant 1}\left|\phi_{n}(x)\right|, \quad M^{\prime}=\sup _{0 \leqslant x \leqslant 1}\left|\phi_{n}^{\prime}(x)\right| .
$$

Since $f$ is $D^{*} B$ a.p. there is a relatively dense set $\{\tau\}$ such that

$$
\rho_{\mathrm{D}^{*} \mathrm{~B}}\left(f_{\tau}, f\right)<\varepsilon\left(M+M^{\prime}\right)^{-1}
$$

for all $\tau \in\{\tau\}$. Let $\tau \in\{\tau\}$. Then writing $F(x)=\int_{0}^{x} f(t) d t$ and $\psi(x)=F(x+\tau)$

- $F(x)$ we have employing Theorem 1.5

(2.2) $\left\|f_{n}(x+\tau)-f_{n}(x)\right\|$

$$
\begin{aligned}
& =\left\|\int_{0}^{1 / n}\{f(t+x+\tau)-f(t+x)\} \phi_{n}(t) d t\right\| \\
& =\|\left[\phi_{n}(t)\{F(t+x+\tau)-F(t+x)\}\right]_{t=0}^{1 / n} \\
& \qquad-\int_{0}^{1 / n}\{F(t+x+\tau)-F(t+x)\} \phi_{n}^{\prime}(t) \| \\
& =\left\|\left[\phi_{n}(t) \psi(x+t)\right]_{t=0}^{1 / n}-\int_{0}^{1 / n} \psi(x+t) \phi_{n}^{\prime}(t) d t\right\| \\
& =\left\|\left[\phi_{n}(t)\{\psi(x+t)-\psi(x)\}\right]_{0}^{1 / n}-\int_{0}^{1 / n}\{\psi(x+t)-\psi(x)\} \phi_{n}^{\prime}(t) d t\right\| \\
& =\left\|\phi_{n}\left(\frac{1}{n}\right)\left\{\psi\left(x+\frac{1}{n}\right)-\psi(x)\right\}-\int_{0}^{1 / n}\{\psi(x+t)-\psi(x)\} \phi_{n}^{\prime}(t) d t\right\|
\end{aligned}
$$


Now let $t \in[0,1 / n]$. Then from (2.1)

$$
\begin{aligned}
\|\psi(x+t)-\psi(x)\| & =\left\|\int_{x}^{x+t}\{f(\xi+t)-f(\xi)\} d \xi\right\| \\
& \leqslant \rho_{\mathrm{D}^{*} \mathrm{~B}}\left(f_{\tau}, f\right)<\varepsilon\left(M+M^{\prime}\right)^{-1} .
\end{aligned}
$$

Hence from (2.2)

$$
\left\|f_{n}(x+\tau)-f_{n}(x)\right\|<M \varepsilon\left(M+M^{\prime}\right)^{-1}+M^{\prime} \varepsilon\left(M+M^{\prime}\right)^{-1}=\varepsilon .
$$

Since $\tau \in\{\tau\}$ is arbitrary, $f_{n}$ is almost periodic for each $n$.

Now since $f$ is uniformly continuous, for every $\varepsilon>0$ there is $\delta>0$ such that

$$
\|f(t+x)-f(x)\|<\varepsilon
$$

whenever $|t|<\delta$. Choose $N$ such that $1 / N \leqslant \delta$. Then when $n \geqslant N$ we have

$$
\begin{aligned}
\left\|f_{n}(x)-f(x)\right\| & =\left\|\int_{0}^{1 / n} f(t+x) \phi_{n}(t) d t-\int_{0}^{1 / n} f(x) \phi_{n}(t) d t\right\| \\
& =\left\|\int_{0}^{1 / n}\{f(t+x)-f(x)\} \phi_{n}(t) d t\right\| \\
& \leqslant \int_{0}^{1 / n}\|f(t+x)-f(x)\| \phi_{n}(t) d t \\
& <\varepsilon .
\end{aligned}
$$

Thus $\left\{f_{n}\right\}$ converges uniformly to $f$. Since each $f_{n}$ is almost periodic, by [1, page 6 , property $\mathrm{V}] f$ is almost periodic.

\section{Mean values and Fourier series}

THEOREM 3.1. If $f$ is $\mathrm{D}^{*} \mathrm{~B}$ a.p. then the mean value

$$
\lim _{T \rightarrow \infty} \frac{1}{T} \int_{0}^{T} f(t) d t=M(f)
$$

exists; further

$$
\lim _{T \rightarrow \infty} \frac{1}{T} \int_{a}^{a+T} f(t) d t=M(f)
$$

uniformly with respect to $a \in \mathbf{R}$.

ProOF. Since

$$
\frac{1}{T} \int_{a}^{a+T} e^{i \lambda t} d t= \begin{cases}1 & \text { if } \lambda=0 \\ \frac{1}{i \lambda T}\left[e^{i \lambda(a+T)}-e^{i \lambda a}\right] & \text { if } \lambda \neq 0\end{cases}
$$


it is clear that

$$
\lim _{T \rightarrow \infty} \frac{1}{T} \int_{a}^{a+T} e^{i \lambda t} d t= \begin{cases}\text { I } & \text { if } \lambda=0 \\ 0 & \text { if } \lambda \neq 0\end{cases}
$$

uniformly with respect to $a \in \mathbf{R}$ and hence for any trigonometric polynomial $P$,

$$
\begin{gathered}
P(t)=\sum_{r=1}^{n} a_{r} e^{i \lambda_{r} t} \quad\left(a_{r} \in \mathbf{X}, \lambda_{r} \in \mathbf{R}\right), \\
\lim _{T \rightarrow \infty} \frac{1}{T} \int_{a}^{a+T} P(t) d t=M(P)
\end{gathered}
$$

exists uniformly with respect to $a \in \mathbf{R}$. Let $\varepsilon>0$ be arbitrary. By Theorem 2.13 there is a trigonometric polynomial $P$ such that $\rho_{\mathrm{D}^{*} \mathrm{~B}}(f, P)<\varepsilon$. Hence

$$
\sup _{\substack{0 \leqslant h \leqslant 1 \\-\infty<x<\infty}}\left\|\int_{x}^{x+h}\{f(t)-P(t)\} d t\right\|<\varepsilon .
$$

Now corresponding to $\varepsilon$ there is $T_{\varepsilon}$ which is independent of $a$, such that

$$
\left\|\frac{1}{T^{\prime}} \int_{a}^{a+T^{\prime}} P(t) d t-\frac{1}{T^{\prime \prime}} \int_{a}^{a+T^{\prime \prime}} P(t) d t\right\|<\varepsilon
$$

for all $T^{\prime}, T^{\prime \prime}>T_{\varepsilon}$.

Set $T_{0}=\max \left[T_{\varepsilon}, 2\right]$ and let $T_{1}, T_{2}>T_{0}$. Then there is a positive integer $N$ such that $N-1<T_{1} \leqslant N$. Putting $h=T_{1} / N$, since $N>2$, we have $\frac{1}{2}<h \leqslant 1$. Now by (3.1) we have

$$
\begin{aligned}
\left\|\frac{1}{T_{1}} \int_{a}^{a+T_{1}}\{f(t)-P(t)\} d t\right\| & =\left\|\frac{1}{N h} \int_{a}^{a+N h}\{f(t)-P(t)\} d t\right\| \\
& =\left\|\frac{1}{N h} \sum_{n=1}^{N} \int_{a+(n-1) h}^{a+n h}\{f(t)-P(t)\} d t\right\| \\
& \leqslant \frac{1}{N h} \sum_{n=1}^{N}\left\|\int_{a+(n-1) h}^{a+n h}\{f(t)-P(t)\} d t\right\| \\
& <\frac{1}{N h} N \varepsilon \\
& <2 \varepsilon
\end{aligned}
$$

since $1 / h<2$. Similarly for $T_{2}>T_{0}$,

$$
\left\|\frac{1}{T_{2}} \int_{a}^{a+T_{2}}\{f(t)-P(t)\} d t\right\|<2 \varepsilon
$$


Since $T_{0} \geqslant T_{\varepsilon}$ we have from (3.2), (3.3) and (3.4) when $T_{1}, T_{2}>T_{0}$,

$$
\begin{aligned}
\| \frac{1}{T_{1}} \int_{a}^{a+T_{1}} f(t) d t & -\frac{1}{T_{2}} \int_{a}^{a+T_{2}} f(t) d t \| \\
\leqslant & \left\|\frac{1}{T_{1}} \int_{a}^{a+T_{1}} f(t) d t-\frac{1}{T_{1}} \int_{a}^{a+T_{1}} P(t) d t\right\| \\
& +\left\|\frac{1}{T_{1}} \int_{a}^{a+T_{1}} P(t) d t-\frac{1}{T_{2}} \int_{a}^{a+T_{2}} P(t) d t\right\| \\
& +\left\|\frac{1}{T_{2}} \int_{a}^{a+T_{2}} f(t) d t-\frac{1}{T_{2}} \int_{a}^{a+T_{2}} P(t) d t\right\| \\
< & 5 \varepsilon .
\end{aligned}
$$

Thus since $\mathbf{X}$ is complete and since $T_{0}$ is independent of $a$,

$$
\lim _{T \rightarrow \infty} \frac{1}{T} \int_{a}^{a+T} f(t) d t=M(f)
$$

exists uniformly with respect to $a \in \mathbf{R}$, completing the proof.

Now if $f$ is $\mathrm{D}^{*} \mathrm{~B}$ a.p. then since $u(x)=e^{-i \lambda x}$ is numerically valued almost periodic function and $u^{\prime}(x)$ is uniformly continuous, by Theorem $2.9 f(x) e^{-i \lambda x}$ is D*B a.p. for all $\lambda \in \mathbf{R}$ and consequently

$$
M\left\{f(x) e^{-i \lambda x}\right\}=\lim _{T \rightarrow \infty} \frac{1}{T} \int_{0}^{T} f(x) e^{-i \lambda x} d x
$$

exists for every $\lambda \in \mathbf{R}$. For a $\mathrm{D}^{*} \mathrm{~B}$ a.p. function $f$ we shall write

$$
a(\lambda)=a(\lambda ; f)=M\left\{f(x) e^{-i \lambda x}\right\} .
$$

Theorem 3.2. If $f$ is $\mathrm{D}^{*} \mathrm{~B}$ a.p. then $a(\lambda ; f)$ differs from the zero element $\theta$ of $\mathbf{X}$ for only an enumerable set of values of $\lambda$.

Proof. Let

$$
F(x)=\int_{0}^{x} f(t) d t
$$

Then for a given $h \in[0,1]$ we have, by integrating by parts by Theorem 1.5 .

$$
\begin{aligned}
\frac{1}{T} \int_{0}^{T}\{f(x+h)-f(x)\} e^{-i \lambda x} d x \\
=\frac{1}{T}\left[\{F(x+h)-F(x)\} e^{-i \lambda x}\right]_{0}^{T} \\
\quad+\frac{i \lambda}{T} \int_{0}^{T}\{F(x+h)-F(x)\} e^{-i \lambda x} d x .
\end{aligned}
$$


Also

$$
\begin{aligned}
\frac{1}{T} \int_{0}^{T}\{f(x+h)-f(x)\} & e^{-i \lambda x} d x \\
& =\frac{1}{T}\left[e^{i \lambda h} \int_{h}^{T+h} f(t) e^{-i \lambda t} d t-\int_{0}^{T} f(t) e^{-i \lambda t} d t\right]
\end{aligned}
$$

Now by Theorem 2.2 the function $F(x+h)-F(x)$ is almost periodic. Let its Fourier coefficients be $\alpha_{h}(\lambda)$. Then applying Theorem 3.1 we get from (3.5) and (3.6), by letting $T \rightarrow \infty$ since $F(x+h)-F(x)$ is bounded,

$$
\left(e^{i \lambda h}-1\right) a(\lambda ; f)=i \lambda \alpha_{h}(\lambda) \text {. }
$$

So, if $\lambda \neq 2 n \pi, n=0, \pm 1, \pm 2, \ldots$

$$
a(\lambda ; f)=\frac{i \lambda}{e^{i \lambda h}-1} \alpha_{h}(\lambda) .
$$

Since $\alpha_{h}(\lambda) \neq \theta$ for at most enumerable number of $\lambda, a(\lambda) \neq \theta$ for these enumerable $\lambda$ and probably for $\lambda=2 n \pi, n=0, \pm 1, \pm 2, \ldots$ Thus $a(\lambda)$ differs from $\theta$ for at most an enumerable set of values of $\lambda$. This completes the proof of the theorem.

Let $\left\{\lambda_{n}\right\}$ be the enumerable set such that $a\left(\lambda_{n}\right) \neq \theta$. Putting $a_{n}=a\left(\lambda_{n}\right)$ we say that $\sum a_{n} e^{i \lambda n x}$ is the Fourier series of $f$ and write

$$
f \sim \sum_{n} a_{n} e^{i \lambda_{n} x} .
$$

LEMMA 3.3. If $f$ is $\mathrm{D}^{*} \mathrm{~B}$ a.p. and $x^{*} \in \mathbf{X}^{*}$ then

$$
x^{*} a(\lambda ; f)=a\left(\lambda ; x^{*} f\right) \text {. }
$$

ProOF.

$$
\begin{aligned}
x^{*} a(\lambda ; f) & =x^{*} M\left\{f(x) e^{-i \lambda x}\right\} \\
& =x^{*} \lim _{T \rightarrow \infty} \frac{1}{T}\left(\mathrm{D}^{*} \mathrm{~B}\right) \int_{0}^{T} f(x) e^{-i \lambda x} d x \\
& =\lim _{T \rightarrow \infty} \frac{1}{T} x^{*}\left(\mathrm{D}^{*} \mathrm{~B}\right) \int_{0}^{T} f(x) e^{-i \lambda x} d x
\end{aligned}
$$

since $x^{*}$ is continuous. Now since a Denjoy-Bochner integrable function is Denjoy-Pettis integrable with integrals equal [5], we have

$$
x^{*}\left(\mathrm{D}^{*} \mathrm{~B}\right) \int_{0}^{T} f(x) e^{-i \lambda x} d x=\left(\mathrm{D}^{*}\right) \int_{0}^{T} x^{*} f(x) e^{-i \lambda x} d x
$$


and hence

$$
\begin{aligned}
x^{*} a(\lambda ; f) & =\lim _{T \rightarrow \infty} \frac{1}{T} \int_{0}^{T} x^{*} f(x) e^{-i \lambda x} d x \\
& =M\left\{x^{*} f(x) e^{-i \lambda x}\right\} \\
& =a\left(\lambda ; x^{*} f\right) .
\end{aligned}
$$

THEOREM 3.4 (Uniqueness Theorem). If two $\mathrm{D}^{*} \mathrm{~B}$ a.p. functions $f$ and $g$ have same Fourier series then

$$
\rho_{\mathrm{D}^{* \mathrm{~B}}}(f, g)=0 .
$$

Proof. Let $x^{*} \in \mathbf{X}^{*}$ be arbitrarily chosen. By Lemma $2.10 x^{*} f$ and $x^{*} g$ are $\mathrm{D}^{*}$ a.p. scalar functions and by Lemma 3.3 they have same Fourier series. As the corresponding theorem of [3] it can be shown that $\rho_{\mathrm{D}^{*}}\left(x^{*} f, x^{*} g\right)=0$, that is,

$$
\sup _{\substack{0 \leq h \leq 1 \\-\infty<x<\infty}}\left|\left(\mathrm{D}^{*}\right) \int_{x}^{x+h}\left\{x^{*} f(t)-x^{*} g(t)\right\} d t\right|=0 .
$$

Now by our previous remark

$$
x^{*}\left(\mathrm{D}^{*} \mathrm{~B}\right) \int_{x}^{x+h}\{f(t)-g(t)\} d t=\left(\mathrm{D}^{*}\right) \int_{x}^{x+h} x^{*}\{f(t)-g(t)\} d t
$$

and hence

$$
\sup _{\substack{0 \leqslant h \leqslant 1 \\-\infty<x<\infty}}\left|x^{*}\left(\mathrm{D}^{*} \mathrm{~B}\right) \int_{x}^{x+h}\{f(t)-g(t)\} d t\right|=0 .
$$

Therefore,

$$
x^{*}\left(\mathrm{D}^{*} \mathrm{~B}\right) \int_{x}^{x+h}\{f(t)-g(t)\} d t=0
$$

for all $x \in \mathbf{R}$ and $h \in[0,1]$. Since $x^{*}$ is arbitrary, by Hahn-Banach Theorem

$$
\left(\mathrm{D}^{*} \mathrm{~B}\right) \int_{x}^{x+h}\{f(t)-g(t)\} d t=\theta
$$

for all $x \in \mathbf{R}$ and $h \in[0,1]$. Therefore

$$
\sup _{\substack{0 \leqslant h \leqslant 1 \\-\infty<x<\infty}}\left\|\int_{x}^{x+h}\{f(t)-g(t)\} d t\right\|=0,
$$

that is,

$$
\rho_{\mathrm{D}^{*} \mathrm{~B}}(f, g)=0
$$




\section{Bochner-Fejer summability of Fourier series}

We shall show that if $f$ be $D^{*} B$ a.p. then the Fourier series of $f$ is Bochner-Fejer summable to $f$ with respect to the metric $\rho_{D^{*} B}$ defined on the space of all $D^{*} B$ a.p. functions. For this purpose we shall use the 'Bochner-Fejer Kernel' and the 'Bochner-Fejer Polynomials' the details of which are discussed in [2, pages 46-50], [1, page 26] and [4, page 153].

Let $f$ be $\mathrm{D}^{*} \mathrm{~B}$ a.p. and let $f(t) \sim \sum a_{k} e^{i \lambda_{k^{t}}}$. Let $\beta_{1}, \beta_{2}, \ldots$ be a basis of the sequence $\left\{\lambda_{k}\right\}$ of the Fourier exponents of $f$. For each positive integer $m$ we consider the Bochner-Fejer Kernel

$$
K_{m}(t)=\sum\left(1-\frac{\left|v_{1}\right|}{(m !)^{2}}\right) \cdots\left(1-\frac{\left|\nu_{m}\right|}{(m !)^{2}}\right) \exp \left(-\frac{i t}{m !} \sum_{k=1}^{m} v_{k} \beta_{k}\right)
$$

and the Bochner-Fejer polynomial for $f$

$$
\begin{aligned}
& \sigma_{m}(t)= \sigma_{m}(t ; f) \\
&=\sum\left(1-\frac{\left|\nu_{1}\right|}{(m !)^{2}}\right) \cdots\left(1-\frac{\left|\nu_{m}\right|}{(m !)^{2}}\right) \\
& \quad \times a\left(\frac{1}{m !} \sum_{k=1}^{m} \nu_{k} \beta_{k} ; f\right) \exp \left(\frac{i t}{m !} \sum_{1}^{m} \nu_{k} \beta_{k}\right),
\end{aligned}
$$

where the first summations in (4.1) and (4.2) extend to all $\nu_{j},\left|\nu_{j}\right| \leqslant(m !)^{2}$, $j=1,2, \ldots, m$, and $a(\lambda ; f)$ in (4.2) is defined by

$$
a(\lambda ; f)=M\left\{f e^{-i \lambda x}\right\} .
$$

If, however, the basis contains a finite number of elements $\beta_{1}, \beta_{2}, \ldots, \beta_{p}$ then we take

$$
\begin{aligned}
\sigma_{m}(t)=\sum(1 & \left.-\frac{\left|\nu_{1}\right|}{(m !)^{2}}\right) \cdots\left(1-\frac{\left|\nu_{p}\right|}{(m !)^{2}}\right) \\
& \times a\left(\frac{1}{m !} \sum_{k=1}^{p} \nu_{k} \beta_{k} ; f\right) \exp \left(\frac{i t}{m !} \sum_{k=1}^{p} \nu_{k} \beta_{k}\right),
\end{aligned}
$$

the summation being extended to $\left|\boldsymbol{\nu}_{j}\right| \leqslant(m !)^{2}, j=1,2 \cdots p$ with similar modification for $K_{m}(t)$. It can be verified that

$$
\sigma_{m}(t ; f)=\lim _{T \rightarrow \infty} \int_{0}^{T} K_{m}(u) f(u+t) d u .
$$

In what follows we need the function

$$
\phi(x, h)=\int_{x}^{x+h} f(t) d t, \quad x \in \mathbf{R}, h \in[0,1] .
$$


For fixed $h \in[0,1]$ this is a function of $x$ alone which is almost periodic by Theorem 2.2. Therefore for arbitrary but fixed $h \in[0,1]$, the $\sigma_{m}(x ; \phi)$ will have the same meaning as given in (4.2).

THEOREM 4.1. Let $f$ be $\mathrm{D}^{*} \mathrm{~B}$ a.p. and let

$$
f(t) \sim \sum a_{k} e^{i \lambda_{k} t}
$$

Then the sequence of trigonometric polynomials $\left\{\sigma_{m}(t ; f)\right\}$ converges to $f$ with respect to the metric $\rho_{\mathrm{D}^{* \mathrm{~B}}}$ as $m \rightarrow \infty$.

We shall complete the proof of the theorem in three lemmas.

LeMma 4.2. If $f$ is $\mathrm{D}^{*} \mathrm{~B}$ a.p. then

$$
\sigma_{m}(x ; \phi) \rightarrow \phi(x, h)
$$

as $m \rightarrow \infty$ uniformly with respect to $x \in \mathbf{R}$ and $h \in[0,1]$ where $\phi(x, h)=$ $\int_{x}^{x+h} f(t) d t$.

Proof. By Theorem $2.2 \phi(x, h)$ is almost periodic in $x \in \mathbf{R}$ uniformly with respect to $h \in[0,1]$. Hence by Lemma 2.3 the Banach valued function $\Phi$ : $\mathbf{R} \rightarrow \mathcal{C}_{\mathbf{X}}[0,1]$ defined by $\Phi(t)=\phi(t, \cdot)$ is almost periodic. If

$$
\Phi(t) \sim \sum b_{n} e^{i \lambda_{n} t}
$$

then $b_{n} \in \mathcal{C}_{\mathrm{X}}[0,1]$ and

$$
b_{n}=\lim _{T \rightarrow \infty} \frac{1}{T} \int_{a}^{a+T} \Phi(t) e^{-i \lambda_{n} t} d t
$$

uniformly with respect to $a$ (see [4, page 146]). By the definition of $\Phi$ we can write

$$
\Phi(t) e^{-i \lambda_{n} t}=\phi(t, \cdot) e^{-i \lambda_{n} t}
$$

and so

$$
\frac{1}{T} \int_{a}^{a+T} \Phi(t) e^{-i \lambda_{n} t} d t=\frac{1}{T} \int_{a}^{a+T} \phi(t, \cdot) e^{-i \lambda_{n} t} d t .
$$

Hence from (4.3)

$$
\lim _{T \rightarrow \infty}\left\|\frac{1}{T} \int_{a}^{a+T} \Phi(t) e^{-i \lambda_{n} t} d t-b_{n}\right\|_{\mathbb{e}_{\mathbf{x}}}=0
$$

uniformly with respect to $a$. That is

$$
\lim _{T \rightarrow \infty} \sup _{0 \leqslant h \leqslant 1}\left\|\frac{1}{T} \int_{a}^{a+T} \phi(t, h) e^{-i \lambda_{n} t} d t-b_{n}(h)\right\|=0
$$


uniformly with respect to $a$. Hence

$$
\lim _{T \rightarrow \infty} \frac{1}{T} \int_{a}^{a+T} \phi(t, h) e^{-i \lambda_{n} t} d t=b_{n}(h)
$$

uniformly with respect to $a$ and $h$. So, $b_{n}(h)$ are the Fourier coefficients of $\phi(t, h)$ and the Fourier exponents of $\Phi(t)$ and $\phi(t, h)$ will remain the same. Now it is proved in [1, page 26] that

$$
\lim _{m \rightarrow \infty} \sigma_{m}(t ; \Phi)=\Phi(t)
$$

uniformly with respect to $t$, where $\sigma_{m}(t ; \Phi)$ is defined as in (4.2) and the limit in (4.4) is taken with respect to the Banach space in which $\Phi(t)$ lies and so (4.4) becomes

$$
\left\|\sigma_{m}(t ; \Phi)-\Phi(t)\right\|_{e_{\mathbf{x}}} \rightarrow 0
$$

as $m \rightarrow \infty$ uniformly with respect to $t$. That is

$$
\sup _{0 \leqslant h \leqslant 1}\left\|\sigma_{m}(t ; \phi)-\phi(t, h)\right\| \rightarrow 0
$$

as $m \rightarrow \infty$ uniformly with respect to $t$. Thus

$$
\sigma_{m}(t ; \phi) \rightarrow \phi(t, h)
$$

as $m \rightarrow \infty$ uniformly with respect to $t$ and $h$.

LEMMA 4.3. If $f$ is $\mathrm{D}^{*} \mathrm{~B}$ a.p. then for each $h \in[0,1]$

$$
\int_{x}^{x+h} \sigma_{m}(t ; f) d t=\sigma_{m}(x ; \phi) .
$$

Integrating (4.2) and using (3.7) the proof can be completed.

LEMMA 4.4. If $f$ is $\mathrm{D}^{*} \mathrm{~B}$ a.p. then $\sigma_{m}(t ; f) \rightarrow f(t)$ as $m \rightarrow \infty$ with respect to the metric $\rho_{\mathrm{D}^{*} \mathrm{~B}}$.

Proof. Let $\phi(x, h)=\int_{x}^{x+h} f(t) d t$. Then by Lemma 4.2

$$
\sigma_{m}(x ; \phi) \rightarrow \phi(x, h)
$$

as $m \rightarrow \infty$ uniformly with respect to $x \in \mathbf{R}$ and $h \in[0,1]$. So,

$$
\sup _{\substack{0 \leq h \leq 1 \\-\infty<x<\infty}}\left\|\sigma_{m}(x ; \phi)-\phi(x, h)\right\| \rightarrow 0
$$

as $m \rightarrow \infty$. Hence by Lemma 4.3

$$
\sup _{\substack{0 \leq h \leq 1 \\-\infty<x<\infty}}\left\|\int_{x}^{x+h} \mathrm{o}_{m}(t ; f) d t-\int_{x}^{x+h} f(t) d t\right\| \rightarrow 0
$$


as $m \rightarrow \infty$. So,

$$
\sup _{\substack{0 \leq h \leq 1 \\-\infty<x<\infty}}\left\|\int_{x}^{x+h}\left\{\sigma_{m}(t ; f)-f(t)\right\} d t\right\| \rightarrow 0
$$

that is,

$$
\rho_{\mathrm{D}^{* \mathrm{~B}}}\left(\sigma_{m}(t ; f), f\right) \rightarrow 0
$$

as $m \rightarrow \infty$. This completes the proof of Theorem 4.1 .

\section{References}

[1] L. Amerio and G. Prouse, Almost periodic functions and functional equations (Von Nostrand Reinhold, New York, 1971).

[2] A. S. Besicovitch, Almost periodic functions (Dover Publications, New York, 1958).

[3] H. Burkill, 'Almost periodicity and non-absolutely integrable functions', Proc. London Math. Soc. (2) 53 (1951), 32-42.

[4] C. Corduneanu, Almost periodic functions (Interscience, New York, 1968).

[5] B. K. Pal, 'Integration by parts formulae for Denjoy-Bochner and Denjoy Pettis integrals', to appear.

[6] D. W. Solomon, Denjoy integration in abstract spaces (Memoirs of the Amer. Math. Soc. 85, 1969).

[7] B. S. Thomson, 'Constructive definition for non-absolutely convergent integrals', Proc. London Math. Soc. (3) 20 (1970), 699-716.

Department of Mathematics

The University of Burdwan

Burdwan 713104, West Bengal

India 